Review

\title{
Inhibition of Late and Early Phases of Cancer Metastasis by NF-KB Inhibitor DHMEQ Derived from Microbial Bioactive Metabolite Epoxyquinomicin: A Review
}

\author{
Yinzhi Lin ${ }^{1}$, Tamami Ukaji ${ }^{2}$, Naoki Koide ${ }^{2}$, and Kazuo Umezawa ${ }^{1, *}$ \\ ${ }^{1}$ Department of Molecular Target Medicine, Aichi Medical University School of Medicine, 1-1 Yazako-Karimata, \\ Nagakute 480-1195, Japan; lin.yinzhi.026@mail.aichi-med-u.ac.jp (Y.L.); umezawa@aichi-med-u.ac.jp (K.U.) \\ ${ }^{2}$ Department of Microbiology and Immunology, Aichi Medical University School of Medicine, 1-1 Yazako-Karimata, \\ Nagakute 480-1195, Japan; ukaji@aichi-med-u.ac.jp (T.U.); koide@aichi-med-u.ac.jp (N.K.) \\ *Correspondence: umezawa@aichi-med-u.ac.jp (K.U.)
}

\begin{abstract}
We previously designed and synthesized dehydroxyepoxyquinomicin (DHMEQ) as an inhibitor of $\mathrm{NF}-\mathrm{KB}$ based on the structure of microbial secondary metabolite epoxyquinomicin C. DHMEQ showed anti-inflammatory and anticancer activity in various in vivo disease models without toxicity. Cell detachment from the primary tumor and subsequent invasion are considered to be early phase of metastasis, while tumor cell attachment to the tissue and secondary tumor formation the late phase. The assay system for late phase was set up with intra-portal-vein injection of pancreatic cancer cells. Administration of DHMEQ was found to inhibit the liver metastasis possibly by decreasing the expression of MMP-9 and IL-8. Also when the pancreatic cancer cells treated with DHMEQ was inoculated into the peritoneal cavity of mice, the metastatic foci formation was inhibited. These results indicate that DHMEQ is likely to inhibit the late phase of metastasis. Meanwhile, we have recently employed three-dimensional (3D) culture of breast cancer cells for the model of early phase metastasis. DHMEQ inhibited the 3D invasion of breast cancer cells without toxicity. In this way, DHMEQ was shown to inhibit the late and early phases of metastasis. Thus, DHMEQ is likely to be useful for the suppression of cancer metastasis.
\end{abstract}

Keywords: epoxyqinomicin; DHMEQ; metastasis; invasion; adhesion; 3D cell culture

\section{Introduction}

Microbial and plant-derived bioactive metabolites are a treasury of organic compounds having various structures and biological activities. On the other hand, many diseases can be explained by abnormality of specific cellular signaling at present. We have isolated various signal transduction inhibitors of low molecular weight from microbial and plant secondary metabolites. These inhibitors include protein-tyrosine kinase inhibitor lavendustin from Streptomyces [1], protein-tyrosine phosphatase inhibitor dephostatin from Streptomyces [2], anti-K-Ras compound aglaiastatin from a plant [3], phosphatidylinositol-specific phospholipase $\mathrm{C}$ inhibitor akaterpin from a marine sponge [4], lipopolysaccharide inactivating compound 
heptadepsin from a bacterium [5], and inhibitor of cancer cell migration migracin from Streptomyces [6]. These are all novel compounds. We have also found that a plant-derived alkaloid conophylline induces pancreatic $\beta$-cell differentiation and protects islets from fibrosis in animals [7]. Thus, these bioactive metabolites should be useful to study the mechanism of diseases, and they can be developed as new therapeutic agents. More recently, we discovered NF- $\mathrm{BB}$ inhibitor dehydroxymethyl-epoxyquinomicin (DHMEQ) by molecular design based on the structure of naturally occurring epoxyquinomicin C. In the present review, we describe inhibition of early and late phases of cancer metastasis by DHMEQ.

\section{Discovery and Mechanism of Action of NF-KB Inhibitor DHMEQ}

In the course of our search for chemical inhibitors of NF- $\kappa B$, we designed and synthesized new NF- $\kappa B$ inhibitors with reference to the structure of epoxyquinomicin $\mathrm{C}$ (Figure 1) [8]. Epoxyquinomicin $\mathrm{C}$ is a weak and useless antibiotic, but it showed no toxicity in animals. Although related compounds such as

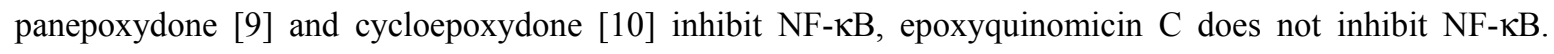
However, after the removal of the protruding hydroxymethyl moiety, the designed compound dehydroxymethylepoxyquinomicin (DHMEQ, Figure 1), did inhibit NF- $\kappa$ B activity. After the synthesis, we found that DHMEQ ameliorated inflammation in a collagen-induced rheumatoid arthritis model in mice when administered by the intraperitoneal (IP) route [8]. Thus, we found a new NF-אB inhibitor active in vivo.

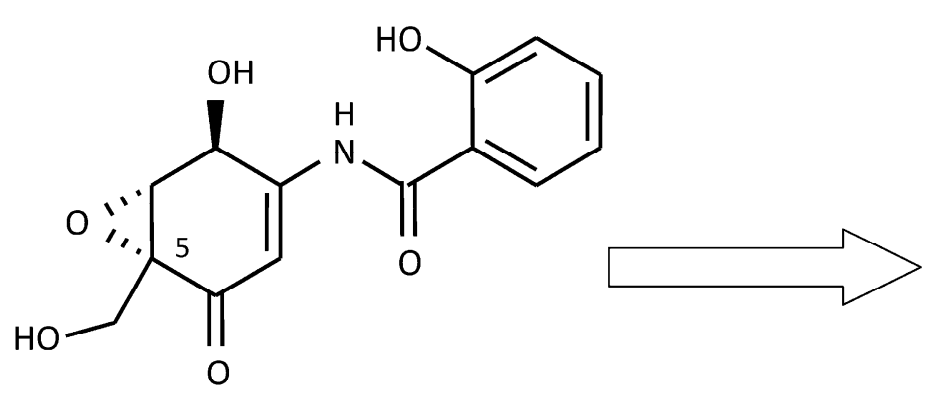

epoxyquinomicin C

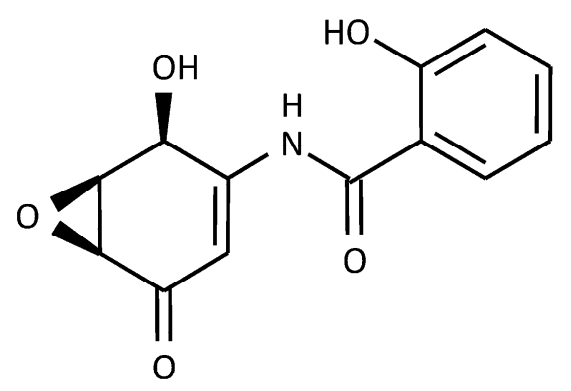

(-)-DHMEQ

Figure 1. Molecular design of (-)-DHMEQ as inhibitor of NF- $\mathrm{\kappa B}$ based on the structure of antibiotic epoxyquinomicin $\mathrm{C}$.

Racemic DHMEQ can be synthesized from a 2,5-dimethoxyaniline in 5 steps [11], and can be separated into each enantiomer practically by lipase [12]. Lipase reacts with racemic dihexanoyl-DHMEQ to give (-)-DHMEQ and monohexanoyl-(+)-DHMEQ that can be easily removed by difference of solubility. (-)-DHMEQ is about 10 times more effective than (+)-DHMEQ in inhibiting NF- $\kappa B$ [11]. (-)-DHMEQ is mainly used for the cellular experiments, and racemic DHMEQ for the animal experiments.

For the mechanism of DHMEQ, we firstly reported that it inhibited the nuclear translocation of NF- $\mathrm{KB}$ 
[13]. However, more recently, we found that DHMEQ directly binds to the Rel-family proteins to inhibit their DNA-binding activity [14]. Inhibition of NF- $\mathrm{BB}$ nuclear translocation is likely to be a result after the inhibition of DNA binding [15]. Rel family proteins are the constituents of NF- $\kappa B$ molecules including p65, Rel B, c-Rel, p50, and p52. (-)-DHMEQ binds to p65 covalently with a 1:1 stoichiometry as revealed by surface plasmon resonance (SPR) and MALDI-TOF mass spectrum (MS) analyses. MS analysis of the chymotrypsin-digested peptide suggested the binding of (-)-DHMEQ to a specific cysteine residue. In the case of p65, DHMEQ only binds to the Cys38 residue, which is located close to the DNA (Figure 2). Observation of the adduct in MALDI-TOF MS suggests that the (-)-DHMEQ-cysteine binding is a covalent one. The formation of (-)-DHMEQ-cysteine covalent binding in the protein was supported by chemical synthesis of the conjugate molecule [16]. Since (-)-DHMEQ binds to the cysteine residue covalently in an NF- $\kappa B$ molecule, the inhibitory effect is irreversible. LPS induces NF- $\kappa B$ activation in $30 \mathrm{~min}$ in a macrophage-like mouse cell line RAW264.7. (-)-DHMEQ was added for only $15 \mathrm{~min}$ and then washed out in the RAW264.7 cell

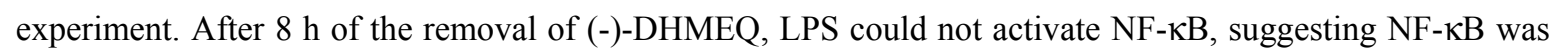
inhibited irreversibly [17].

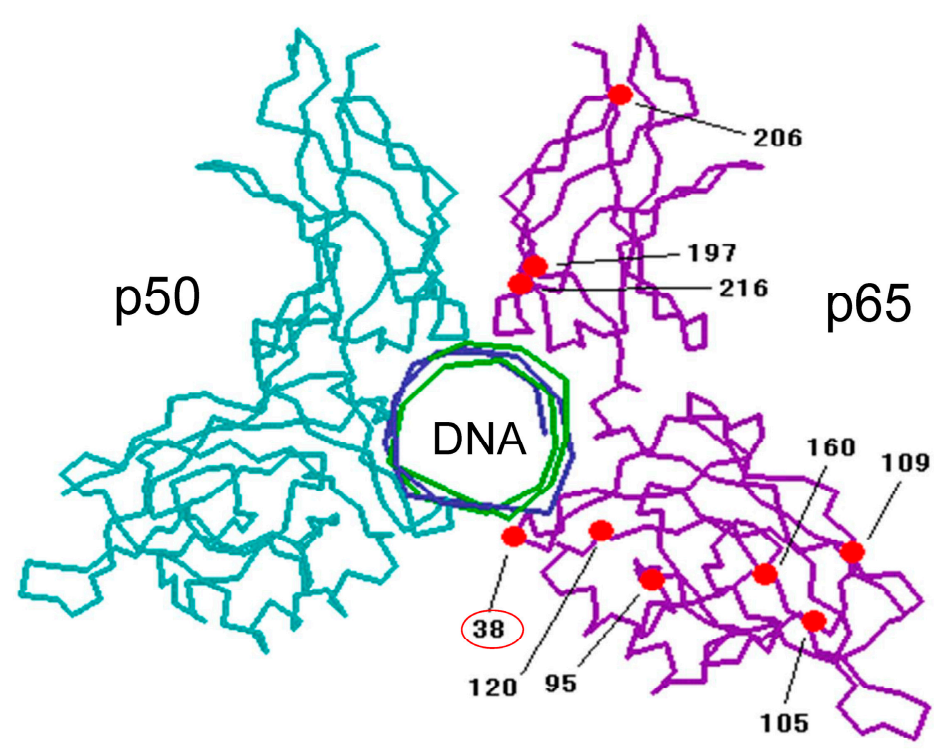

Figure 2. (-)-DHMEQ binds to Cys38 in p65. Cysteine residues are shown in red.

All Rel family proteins possess specific cysteine residues essential for their DNA binding. (-)-DHMEQ binds to p65, cRel, RelB, and p50, but not to p52 at the specific cysteine residues. (-)-DHMEQ is the first $\mathrm{NF}-\kappa \mathrm{B}$ inhibitor shown to covalently bind to a specific cysteine. We have also found that (-)-DHMEQ inhibits not only DNA-binding of RelB, but also its interaction to importin [18]. It also induces instability of RelB.

Thus, (-)-DHMEQ specifically binds to a cysteine residue in both the canonical and the noncanonical NF- $\mathrm{KB}$ components $[14,18]$. It is likely that DHMEQ can enter into a specific pocket via a key and lock mechanism to bind limitedly to the limited cysteine residue. These findings may explain the highly selective $\mathrm{NF}-\kappa \mathrm{B}$ inhibition and the low toxic effect of DHMEQ in cells and in animals. 


\section{Therapeutic Activity of DHMEQ on Inflammatory and Neoplastic Disease models}

DHMEQ was used for many animal experiments to suppress various inflammation and neoplastic diseases and to study the mechanism of diseases. Recent results on its anti-inflammatory and anticancer activities are described below.

DHMEQ is now being developed as an ointment drug for the treatment of atopic dermatitis and other severe skin inflammations. In the genetic atopic dermatitis model, DHMEQ ointment showed similar or stronger anti-inflammatory activities compared with betamethasone and tacrolimus ointments [19]. Accumulation of mast cells was inhibited by DHMEQ ointment in this model. Later DHMEQ was found to inhibit MMP-2 expression and cellular invasion of mouse primary culture mast cells treated with DNP antigen and IgE [20]. More recently, DHMEQ ointment was shown to suppress development of chemically induced atopic dermatitis-like lesions in BALB/c mice [21]. Atopic dermatitis-like lesions were chronically induced by the repetitive and alternative application of 2,4-dinitrochlorobenzene (DNCB) and oxazolone (OX) on ears. The mice were then externally treated with DHMEQ ointment. DHMEQ inhibited ear swelling and relieved clinical symptoms of the atopic dermatitis-like lesions induced by DNCB/OX in BALB/c mice. Histopathology examination illustrated that it significantly decreased DNCB/OX-induced epidermal thickness, the infiltration of inflammatory cells, and the count of mast cells. The elevated level of IgE in serum and the mRNA levels of IL-4 and IL-13 in the ear tissues were also suppressed by DHMEQ.

Amniotic apoptosis is essential for the onset of delivery. On the other hand, too early amniotic apoptosis causes baby loss. Amniotic apoptosis is induced by the TNF- $\alpha$ pathway via the TNF- $\alpha$ receptor 1 expressed in the amniotic epithelial cells. Activated macrophages may cause amniotic apoptosis mediated by TNF- $\alpha$ and NO expression and production. DHMEQ inhibited TNF- $\alpha$ and iNOS expressions in pregnant mice to inhibit amniotic apoptosis [22].

DHMEQ ameliorates organ transplantation in many ways acting as an immunosuppressant. Recently, DHMEQ was found to ameliorate graft-versus-host disease (GVHD) in allogeneic bone marrow transplantation [23]. GVHD is a crucial mortality factor in allogeneic bone marrow transplantation. DHMEQ suppressed GVHD, resulting in an improved mortality rate in a mouse allogeneic bone marrow transplantation model. Bone marrow cells from C57BL/6 mice (B6 mice) were transplanted into lethally irradiated BALB/c mice. Two weeks later, spleen cells from B6 mice were transplanted into the irradiated BALB/c mice. From one week after the injection of spleen cells, when the mice started to show GVHD, the mice were also IP-injected daily with DHMEQ or vehicle for 4 weeks. By 80 days after the transplantation, 6/14 of the vehicle-injected mice (43\%) had died because of GVHD, whereas all DHMEQ-injected mice survived this observation period and developed milder GVHD than the vehicle-injected mice. These findings suggest that administration of DHMEQ would become a new strategy for preventing fatalities from GVHD. 
Recently, it was reported that IP administration of DHMEQ ameliorates dinitrobenzene sulfonic acid (DNBA)-induced colitis in rats [24]. IP administration of DHMEQ also inhibited dextran-sulfate-sodium-induced colitis in rats [25].

$\mathrm{NF}-\mathrm{KB}$ in cancer cells often induces immunosuppression in cancer patients to facilitate the cancer progression. DHMEQ inhibited the NF- $\mathrm{KB}$-dependent immunosuppression in ovarian carcinoma in animal experiment [26]. Although T-cell immunity is considered to be involved in the prognosis of epithelial ovarian cancer patients, immunosuppressive conditions often occurs to hamper antitumor immune responses. In epithelial ovarian cancer patients, increase of plasma IL-6, and IL-8 were observed. DHMEQ was found to inhibit the production of IL-6 and IL-8 in epithelial ovarian cancer cell lines. IP administration of DHMEQ to nude mice implanted with human epithelial ovarian cancer cells resulted in the restoration of T-cell stimulatory activity of murine dendritic cells along with the reduction of tumor accumulation. Inhibition of $\mathrm{NF}-\mathrm{KB}$ in tumor-bearing mice also enhanced antitumor effects of transferred murine naive $\mathrm{T}$ cells. Thus, $\mathrm{NF}-\mathrm{KB}$ is involved in the immunosuppression induced by human epithelial ovarian cancer cells, and its inhibitor such as DHMEQ may restore anticancer immune responses.

DHMEQ inhibits cancer progression in various animal models. Cholangiocarcinoma is one of the most difficult cancers to treat, and there is no effective chemotherapeutic regimen at present. Seubwai and coworkers reported that IP administration of DHMEQ inhibited the growth of cholangiocarcinoma in mice [27]. Normal bile duct epithelia rarely expressed NF- $\mathrm{kB}$ subunits such as $\mathrm{p} 50, \mathrm{p} 52$ and $\mathrm{p} 65$, whereas all cholangiocarcinoma patient tissues over-expressed these NF-KB subunits. DHMEQ increased cell apoptosis by decreasing the expressions of anti-apoptotic proteins such as Bcl-2 and XIAP. Moreover, DHMEQ effectively reduced tumor size in cholangiocarcinoma-inoculated mice.

Ito and coworkers investigated the anticancer effect of DHMEQ in CDDP-resistant bladder cancer cells [28]. Invasive bladder carcinoma cell line T24 and its CDDP-resistant cell line T24PR were used. The NF- KB activity was stronger in T24PR cells than in T24 cells. Lowered cell viability and strong induction of apoptosis were observed by treatment with DHMEQ alone in T24PR cells compared with T24 cells. T24PR cells did not show dramatic cross-resistance to paclitaxel in the in vitro study. They next examined whether the combination of DHMEQ with paclitaxel could enhance the therapeutic effect of the paclitaxel treatment in T24PR tumors. Using mouse xenograft models, the mean volume of tumors treated with the combination of DHMEQ and paclitaxel was significantly smaller than those treated with paclitaxel alone. Thus, DHMEQ showed anticancer activity alone, and also increased the sensitivity to paclitaxel.

\section{Contribution of NF-KB to Cancer Metastasis}

Meanwhile cancer cell proliferation and metastasis are often influenced by the microenvironment. The microenvironment consists of various cell types including endothelial cells forming lymph and blood vessels, 
fibroblasts, and bone marrow derived cells such as macrophages, neutrophils, and mast cells. The tumor microenvironment contributes to tumor progression by secretion of growth factors, cytokines and chemokines. These cytokine and chemokine transcriptions are often dependent on NF- $\kappa$ B. Besides providing growth factors and cytokines, the tumor microenvironment promotes tumor progression by extracellular matrix (ECM) degradation mainly by matrix metallo-proteases (MMPs). Up-regulated MMP secretion is found in many cancer cells. MMP secretions were shown to associate with enhanced cell proliferation, migration, angiogenesis, metastasis and poor survival. The functions of MMPs are (i) cleaving cell adhesion molecules such as E-cadherin, (ii) the degradation of ECM proteins, and (iii) the processing and activation of cytokines and growth factors. Even natural immune cells such as macrophages of the tumor microenvironment often execute a tumor-promoting rather than a tumor-suppressing role. It is well established that chronic infections and inflammation frequently lead to cancer development and enhancement of tumor progression. Interestingly, $\mathrm{NF}-\mathrm{\kappa B}$ has been identified as a key activating signaling pathway in both cancer cells and tumor-associated immune cells. Expression of cytokines such as IL-1, IL-6, TNF- $\alpha$ and many MMPs such as MMP-9, MMP-13, and MT1-MMP is dependent on NF- $\mathrm{KB}$. These protein expressions and productions cause inflammation in the tissue, and cancer cell proliferation and metastasis.

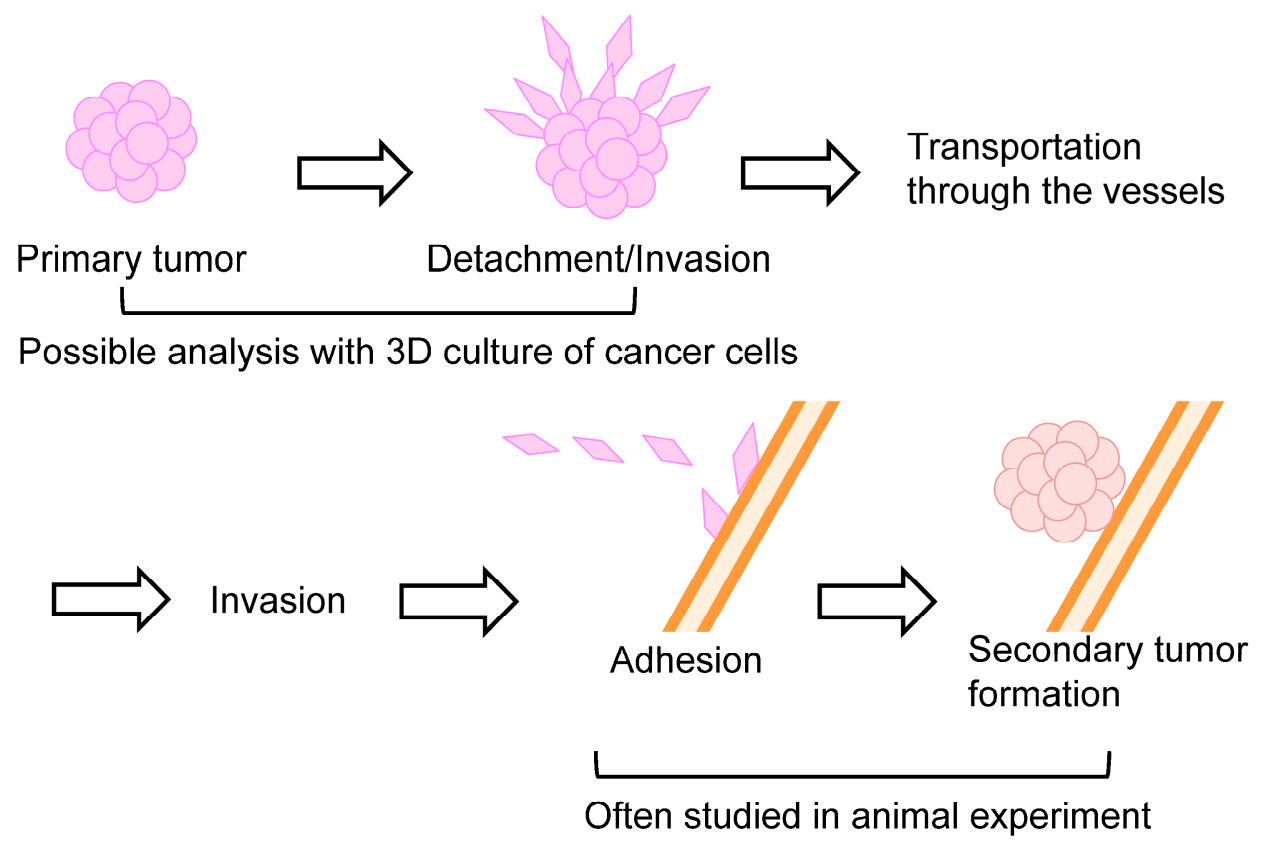

Figure 3. Several steps of metastasis. The early phase including cell detachment and invasion can be studied by 3D invasion of cultured cells, and later phase including invasion and attachment is commonly studied by injection of cancer cells into the transporting veins in animal experiments. 


\section{Inhibition of Late and Early Phases of Metastasis by DHMEQ}

The process of cancer metastasis consists of cell detachment from the primary tumor, invasion, transportation by blood or lymphatic vessels, invasion, attachment, and formation of secondary tumor (Figure 3). Typical in vivo metastasis model is lung metastasis of human cancer cells in rodents. In this model, the cancer cells are inoculated into the tail vein. We previously found that lung metastasis of Yoshida ascites hepatoma AH7974 cells was inhibited by intraperitoneal administration of protease inhibitor leupeptin [29]. On the other hand, tumors in pancreas often metastasize into the liver. Suzuki and coworkers investigated the effects of DHMEQ on the inhibition of liver metastasis of human pancreatic cancer in a mouse model [30]. Nude mice were xenografted by intra-portal-vein injection with human pancreatic adenocarcinoma AsPC-1 cells via laparotomy (Figure 4). Mice were treated with DHMEQ and gemcitabine, alone or in combination. DHMEQ alone inhibited the metastasis. The combination of gemcitabine and DHMEQ showed a stronger antitumor effect than either monotherapy. Apoptosis induction in the metastatic foci was most prominent in the DHMEQ and gemcitabine group. Significant reductions in the numbers of neovessels were also seen in the DHMEQ and/or gemcitabine groups. On the other hand, cell growth inhibition assays revealed no synergistic effect of combination therapy, although each monotherapy had an individual cytotoxic effect. For the mechanism, DHMEQ alone markedly down-regulated expressions of MMP-9 and interleukin (IL)-8 in metastatic foci. These results demonstrate that DHMEQ can exert anticancer effects by inhibiting angiogenesis and tumor cell invasion. Combination therapy with DHMEQ and gemcitabine also showed potential efficacy. Thus, DHMEQ may be useful for the treatment of advanced pancreatic cancer. DHMEQ was given to animals by IP administration in this study. In case of intraperitoneal administration, DHMEQ is considered to act only in the peritoneal cavity suppressing the activity of inflammatory cells in the peritoneal cavity [31]. As a result, the blood level of inflammatory cytokines is lowered to inhibit inflammation and cancer progression indirectly [32]. 


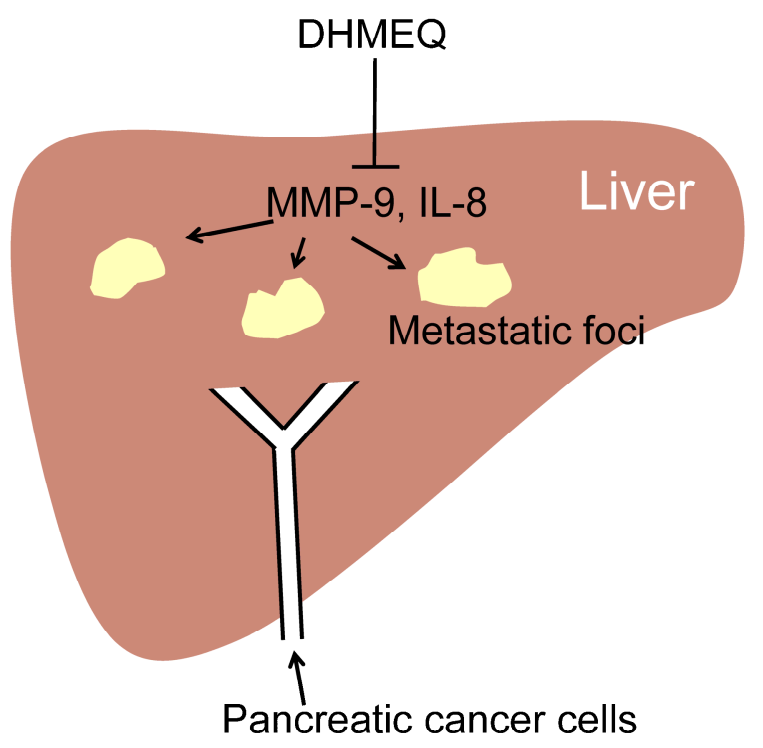

Figure 4. Inhibition of pancreatic cancer cell metastasis in liver by intraperitoneal administration of DHMEQ in mice.

$\mathrm{NF}-\kappa \mathrm{B}$ is also involved in resistance to anoikis, a special type of apoptosis induced when cells are detached from the extracellular matrix or other cells. Anoikis resistance is related to the metastatic abilities of tumor cells. Sato and coworlers employed DHMEQ to investigate anoikis induction and peritoneal metastasis suppression following the cellular NF- $\mathrm{KB}$ inhibition [33]. Pancreatic cancer AsPC-1 cells were photo-marked by Gluc, a secretory form of luciferase for in vivo experiments. DHMEQ induced anoikis in AsPC-1-Gluc cells as measured by the cell survival assays and flow cytometry. DHMEQ inhibited cellular NF- $\mathrm{BB}$ and the subsequent expressions of anti-apoptotic molecules. In a murine xenograft model, anoikis-resistant PC cell lines tended to metastasize to the peritoneum more than anoikis-sensitive cells, suggesting a correlation between anoikis sensitivity and peritoneal metastasis. DHMEQ inhibited peritoneal metastasis of AsPC-1-Gluc cells. They monitored metastasis inhibition by ex-vivo chemiluminescent detection of Gluc secreted from tumor cells into murine plasma and by in vivo imaging. Thus, DHMEQ inhibited peritoneal dissemination by preventing anoikis resistance of PC cells. These results suggest that DHMEQ can inhibit the late phase of metastasis.

The three-dimensional (3D) culture of cancer cells provides an environmental condition closely related to the condition in vivo. Recently, 3D culture of cancer cells has been developed with round bottom well. The cancer cells grow in soft agar forming spheroid colonies. We employed human breast carcinoma MDA-MB-231 cells for 3D culture. Then, I have found that 3D invasion of MDA-MB-231 cells from the colony is just like illustration of early phase of metastasis, as shown in (Figure 5) [34]. I thought it would be an ideal model for the early phase of metastasis, including the detachment and invasion of cancer cells from the primary tumor. Then, we studied the inhibitory activity of DHMEQ on the $3 \mathrm{D}$ invasion of breast 
carcinoma cells. MDA-MB-231 cells showed the most active invasion from spheroid among the cell lines tested. DHMEQ inhibited the 3D invasion of cells at the 3D-nontoxic concentrations. The PCR array analysis using RNA isolated from the 3D on-top cultured cells indicated that MMP-2 expression is lowered by DHMEQ. Knockdown of MMP-2 inhibited the invasion. DHMEQ was shown to inhibit the promoter activity of MMP-2 in the reporter assay. Thus, DHMEQ was shown to inhibit NF-кB/MMP-2-dependent cellular invasion in 3D-cultured MDA-MB-231 cells, suggesting that DHMEQ would inhibit the early phase of metastasis.

Day 3

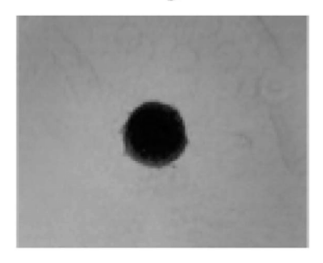

Day 5

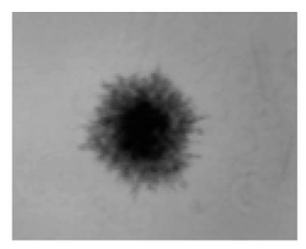

$\mathrm{NF}-\kappa \mathrm{B}$

DHMEQ

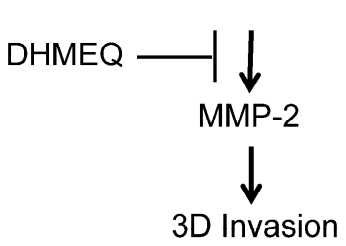

Figure 5. Time-course of 3D invasion in breast carcinoma MDA-MB-231 cells. (Modified from the figure in Reference 34). The cells were cultured in the 96-well round bottom well. DHMEQ inhibited 3D invasion by the decrease of MMP-2 expression.

\section{Conclusions and Future Prospective}

The late phase of metastasis is commonly studied by injection of cancer cells into the transporting blood vessels. DHMEQ inhibited traditional in vivo metastasis models that are for the late phase of metastasis. On the other hand, we first employed 3D culture of human breast cancer MDA-MB-231 cells as a model of early phase of metastasis. Meanwhile DHMEQ derived from microbial bioactive metabolite epoxyquinomicin $\mathrm{C}$ is a specific inhibitor of NF-KB. DHMEQ inhibited the models of both the late and early phases' metastasis (Figure 6). Since DHMEQ is nontoxic in animals, it may become a useful anti-metastatic agent. 


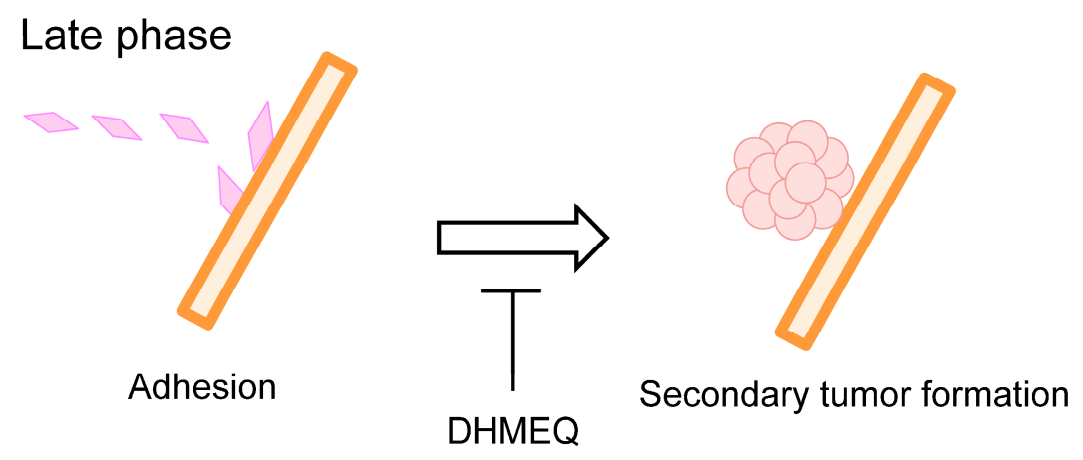

Early phase

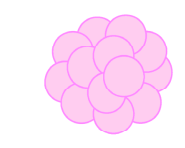

Primary tumor
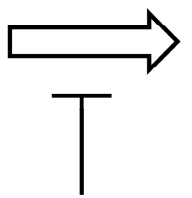

DHMEQ

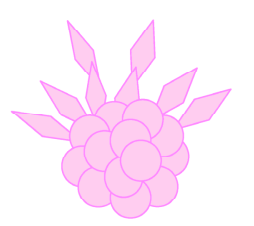

Detachment / Invasion

Figure 6. DHMEQ would inhibit both late and early phases of metastasis.

Acknowledgments: This work was financially supported in part by JSPS Kakenhi Grant Number 17K01967, the Anti-HBV project fund from Japan Agency for Medical and Research Development (AMED), and the Aichi Medical University Research Unit Fund.

Conflicts of Interest: The authors declare no conflict of interest.

\section{References}

1. Onoda T.; Inuma H.; Sasaki Y.; Hamada M.; Isshiki K.; Naganawa H..; Takeuchi T.; Tatsuta K.; Umezawa K. Isolation of a novel tyrosine kinase inhibitor, lavendustin A, from Streptomyces griseolavendus. J. Nat. Prod. 1989, 52, 1252-1257.

2. Imoto M.; Kakeya H.; Sawa T.; Hayashi C.; Hamada M.; Takeuchi T. and Umezawa K. Dephostasin, a novel protein tyrosine phosphatase inhibitor produced by Streptomyces. I. Taxonomy, isolation, and characterization. $J$. Antibiot. 1993, 46: 1342-1346.

3. Ohse T.; Ohba S.; Yamamoto T.; Koyano T. and Umezawa K. Cyclopentabenzofuran lignan protein synthesis inhibitors from Aglaia odorata. J. Nat. Prod. 1996, 59 : 650-652.

4. Fukami A.; Ikeda Y.; Kondo S.; Naganawa H.; Takeuchi T.; Furuya S.; Hirabayashi Y.; Shimoike K.; Hosaka S.; Watanabe Y. and Umezawa K. Akaterpin, a novel bioactive triterpene from the marine sponge Callyspongia sp. Terrahedron Lett. 1997, 38, 1201-1202.

5. Ohno O.; Ikeda Y.; Sawa R.; Igarashi M.; Kinoshita N.; Suzuki Y.; Miyake K. and Umezawa K. Isolation of heptadepsin, a novel bacterial cyclic depsipeptide that inhibits lipopolysaccharide activity. Chemistry \& Biology. 2004, 11, 1059-1070.

6. $\quad$ Arai Y.; Iinuma H.; Ikeda Y.; Igarashi M.; Hatano K.; Kinoshita N.; Ukaji T.; Simizu S. and Umezawa K. Migracins A and B, new inhibitors of cancer cell migration, produced by Streptomyces sp. J. Antibiot. 2013, 66, 225-230.

7. Umezawa K.; Kojima I.; Simizu S.; Lin Y.Z; Fukatsu H.; Koide N.; Nakade Y. and Yoneda M. Therapeutic a activity of plant-derived alkaloid conophylline on metabolic syndrome and neurodegenerative disease models. Human Cell. (in press), 2017 Dec 16. DOI: 10.1007/s13577-017-0196-4.

8. Matsumoto N, Ariga A, To-e S, Nakamura H, Agata N, Hirano S, Inoue J, Umezawa K. Synthesis of NF- $\mathrm{kB}$ activation inhibitors derived from epoxyquinomicin C. Bioorg Med Chem Lett. 2000, 10, 865-869.

9. Erkel G, Anke T, Sterner O. Inhibition of NF- $\mathrm{KB}$ activation by panepoxydone. Biochem Biophys Res Commun. 1996, 226, 214-221.

10. Gehrt A, Erkel G, Anke T, Sterner OA. Cycloepoxydon: 1-hydroxy-2-hydroxymethyl-3-pent-1-enylbenzene1-hydroxy-2-hydroxymethyl-3-pent-1, 3-dienylbenzene, new inhibitors of eukaryotic signal transduction. J Antibiot. 1998, 51, 455-463.

11. Suzuki Y, Sugiyama C, Ohno O, Umezawa K. Preparation and biological activities of optically active 
dehydroxymethylepoxyquinomicin, a novel NF-кB inhibitor. Tetrahedron. 2004, 60, 7061-7066.

12. Hamada M.; Niitsu Y.; Hiraoka C.; Kozawa I.; Higashi T.; Shoji M.; Umezawa K.; Sugai T. Chemoenzymatic synthesis of $(2 \mathrm{~S}, 3 \mathrm{~S}, 4 \mathrm{~S})$-form, the physiologically active stereoisomer of dehydroxymethylepoxyquinomicin (DHMEQ), a potent inhibitor on NF-אB. Tetrahedron. 2010, 66, 7083-7087.

13. Ariga A, Namekawa J, Matsumoto $N$, Inoue $J$, Umezawa K. Inhibition of TNF- $\alpha$-induced nuclear translocation and activation of NF- $\kappa$ B by dehydroxymethyl-epoxyquinomicin. $J$ Biol Chem. 2002, 277, 27625-27630.

14. Yamamoto M, Horie R, Takeiri M, Kozawa I, Umezawa K. Inactivation of nuclear factor kappa B components by covalent binding of (-)-dehydroxymethylepoxyquinomicin to specific cysteine residues. J Med Chem. 2008, 51, $5780-5788$

15. Horie K.; Ma J. and Umezawa K. Inhibition of canonical NF- $\kappa B$ nuclear localization by (-)-DHMEQ via impairment of DNA binding. Oncology Res. 2015, 22, 105-115.

16. Kozawa I, Kato $\mathrm{K}$, Teruya $\mathrm{T}$, Suenaga $\mathrm{K}$, Umezawa $\mathrm{K}$. Unusual intramolecular $\mathrm{N} \rightarrow \mathrm{O}$ acyl group migration occurring during conjugation of (-)-DHMEQ with cysteine. Bioorg Med Chem Lett. 2009, 19, 5380-5382.

17. Shimada C, Ninomiya Y, Suzuki E, Umezawa K. Efficient cellular uptake of the novel NF-kB inhibitor (-)-DHMEQ and irreversible inhibition of NF-KB in neoplastic cells. Oncology Research. 2010, 18, 529-535.

18. Takeiri M, Horie K, Takahashi D, Watanabe M, Horie R, Simizu S, Umezawa K. Involvement of DNA binding domain in the cellular stability and importin affinity of NF-kB component RelB. Org Biomol Chem. 2012,10, 3053-3059.

19. Hamasaka A.; Yoshioka N.; Abe R.; Kishino S.; Umezawa K.; Ozaki M.; Todo S. and Shimizu H. Topical application of DHMEQ improves allergic inflammation via NF- $\mathrm{KB}$ inhibition. Journal of Allergy and Clinical Immunology. 2010, 126, 400-403.

20. Noma N.; Asagiri M.; Takeiri M.; Ohmae S.; Takemoto K.; Iwaisako K.; Simizu S. and Umezawa K. Inhibition of MMP-2-mediated mast cell invasion by NF- $\mathrm{KB}$ inhibitor DHMEQ in mast cells. International Achieves of Allergy and Immunology. 2015, 166, 84-90.

21. Jiang X.; Wei B.; Lan Y.; Dai C.; Gu Y.; Ma J.; Liu X.; Umezawa K. and Zhang Y. External application of NF- $\mathrm{\kappa B}$ inhibitor DHMEQ suppresses development of atopic dermatitis-like lesions induced with DNCB/OX in BALB/c mice. Immunopharmacology and Immunotoxicology. 2017, 39(3), 157-164.

22. Kobayashi K, Umezawa K, Yasui M. Apoptosis in mouse amniotic epithelium is induced by activated macrophages through the TNF receptor type 1/TNF pathway. Biology of Reproduction. 2011, 84, 248-254.

23. Yamanouchi S.; Adachi Y.; Shimo T.; Umezawa K.; Okigaki M.; Tsuji S.; Li M.; Takaya J.; Kuge T.; Ikehara S.; Kaneko K. A nuclear factor- $\mathrm{KB}$ inhibitor, dehydroxymethylepoxyquinomicin, ameliorates GVHD in allogeneic bone marrow transplantation. Immunobiology. 2015, 220, 1059-1066.

24. El-Salhy M. and Umezawa K. Effects of AP-1 and NF-kappa B inhibitors on colonic endocrine cells in rats with TNBS-induced colitis. Molecular Medicine Reports. 2016, 14, 1515-1522.

25. El-Salhy $M$. and Umezawa K. Anti-inflammatory effects of novel AP-1 and NF-kB inhibitors in dextran-sulfate-sodium-induced colitis in rats. International J. Mol. Med. DOI: 10.3892/ijmm. 2016, 37(6), 14571464.

26. Nishio H.; Yaguchi T.; Sugiyama J.; Sumimoto H.; Umezawa K.; Iwata T.; Susumu N.; Fujii T.; Kawamura N.; Kobayashi A.; Park J.H.; Aoki D.; Kawakami D. Immunosuppression through constitutively activated NF-kB signaling in human ovarian cancer and its reversal by a NF-кB inhibitor. British J. Cancer. 2014, 110, 2965-2974.

27. Seubwai W.; Kraiklang R.; Vaeteewoottacharn K.; Umezawa K.; Okada S.; Wongkham S. Aberrant expression of NF- $\mathrm{kB}$ in liver fluke associated cholangiocarcinoma: implications for targeted therapy. PLOS ONE. 2014, 9 (8), e106056. DOI: 10.1371/journal.pone.0106056.

28. Ito Y.; Kikuchi E.; Tanaka N.; Kosaki T.; Suzuki E.; Mizuno R.; Shinojima T.; Miyajima A.; Umezawa K. and Oya M. Down-regulation of NF kappa B activation is an effective therapeutic modality in acquired platinum-resistant bladder cancer. BMC Cancer. 2015,15,324. DOI: 10.1186/s12885-015-1315-9.

29. Saito D.; Sawamura M.; Umezawa K.; Kanai Y.; Furihata C.; Matsushima T. and Sugimura T. Inhibition of experimental blood-borne lung metastasis by protease inhibitors. Cancer Res. 1980, 40, 2539-2542.

30. Suzuki K.; Aiura K.; Matsuda S.; Itano O.; Takeuchi O.; Umezawa K. and Kitagawa Y. Combined effect of dehydroxymethylepoxyquinomicin and gemcitabine in a mouse model of liver metastasis of pancreatic cancer. Clinical \& Experimental Metastasis 2013, 30, 381-392.

31. Sosinnska P.; Macckowiak B.; Staniszewski R.; Umezawa K.and Breborowicz A. Inhibition of NF- $\kappa B$ with dehydroxyepoxiquinomicin modifies function of human peritoneal mesothelial cells. American Journal of Translational Research 2016, 8, 5756-5765.

32. Umezawa K. Peritoneal NF- $\mathrm{\kappa B}$ as a Possible molecular target for suppression of various cancers and inflammation. (Review) Forum of Immunopathological Diseases and Therapeutics 2013, 4, 63-77.

33. Sato M.; Nakanishi K.; Haga S.; Fujiyoshi M.; Baba M.; Mino K.Y.; Niwa H.; Yokoo H.; Umezawa K.; Ohmiya Y.; Kamiyama T.; Todo S.; Taketomi A.; Ozaki M. Anoikis induction and inhibition of peritoneal metastasis of pancreatic cancer cells by a nuclear factor-kappa B inhibitor, (-)-DHMEQ. Oncology Res. 2014, 21, 333-343.

34. Ukaji T.; Lin Y.Z; Okada S. and Umezawa K. Inhibition of MMP-2-mediated cellular invasion by NF- $\mathrm{BB}$ inhibitor DHMEQ in 3D culture of breast carcinoma MDA-MB-231 cells: A model for early phase of metastasis. Biochem. Biophys. Res. Commun. 2017, 485, 76-81. 